\title{
Screening of Linseed Germplasm for Resistance/Tolerance against Fusarium oxysporum F Sp. Lini (Bolley) Disease
}

Mohit Kumar $^{1 *}$, Tripathi UK ${ }^{1}$, Ajay Tomer ${ }^{2}$, Pankaj Kumar ${ }^{2}$ and Anchal Singh ${ }^{3}$

${ }^{1}$ Department of Plant Pathology and Entomology, Chandra Shekhar Azad University of Agriculture and Technology, Kanpur, India ${ }^{2}$ Department of Plant Pathology and Entomology, Sardar Vallabhbhi Patel University of Agriculture and Technology, Meerut, India ${ }^{3}$ Department of Plant Pathology and Entomology, Amity University, Noida, UP, India

\begin{abstract}
Absence of resistance/tolerance against diseases and insect pests in linseed (Linum usitatissimum L.) varieties is one of the main reasons for their low yield in India. During the summer season, Fusarium oxysporum $\mathrm{f} s p$. lini epidemic damage the crop in most of the linseed seed growing area. For the purpose of identifying resistance/ tolerance in linseed germplasm, a disease screening in trial, comprising of 200 test entries was developed. Screening was done under natural conditions in 2012 at C.S.A. University of agriculture and technology Kanpur. Out of 200 germplasm, 116 resistances, 51 moderately resistant, 30 moderately susceptible, 3 susceptible and 1 germplasm highly susceptible were found.
\end{abstract}

Keywords: Resistance; Germplasm; Screening; Fusarium oxysporum f sp. lini.

\section{Introduction}

Cultivated Flax (Linum usitatissimum L.), commonly known as 'linseed' or 'alsi' is a extensively cultivated oilseed crop. It is one of the oldest cultivated crops, grown either for fiber or oil. Fiber is obtained from flax straw and used to make paper and linen, while the oil is attained from the seeds and has been used in the production of oil which is used in the industries as well as domestically [1]. In industries oil is used for the manufacturing of paints, varnish, oil cloth, linoleum, printing ink etc. The oil cake is used as manure to maintain the fertility level as well as agent against soil born pathogen. The productivity of this important oil seed crop is very low in India. Among various factors responsible for lowering down its yield, diseases are important one. The most important disease which stand out is, wilt caused by Fusarium oxysporum $\mathrm{f}$ sp. lini. Causes significant yield loss [2]. Besides loss in seed yield, it reduces the quality of the seed also [3]. However the resistance among varieties can differ due to the variability of pathogen races in different geographical regions with varying temperature and environmental conditions $[3,4]$.

\section{Material and Methods}

In case of soil-borne disease such as wilt, use of resistant germplasm varieties is the most economic and ecofriendly method of management. Keeping in view the importance of this method, 'the study was conducted under field condition in the wilt sick plot of Nawabganj Research farm. A set of 200 germplasm varieties of linseed received from project coordinating unit (Linseed) (P.C. unit) situated at C.S. Azad University of Agriculture and Technology, Kanpur were screened for resistance against the pathogen. Each germplasm variety under test was sown in 2.5 meters long, single row, $25 \mathrm{~cm}$ apart, in rod row design A highly susceptible variety (Chambal) was planted after every $6^{\text {th }}$ row of test entries. Fertilizer application, irrigation, weeding and other intercultural operation were done as per the normal recommended practice. The total numbers of plants in each row were counted for initial plant stand wilting of plants was carefully monitored right after emergence of seeding to crop maturity. The final wilt incidence was calculated by deducting the number of plants survived from the initial crop stand. The percentage of wilting was calculated using the following formula:

$$
\text { Percentage wilt incidence }=\frac{\text { Number of wilted plants }}{\text { Total plant population }} \times 100
$$

The following rating scale was used for grouping of resistance and susceptibility to categories the germplasm/varieties (Table 1).

\section{Result and Discussion}

The linseed germplasm/varieties against F. oxysporum f. sp. lini interaction presented in Table 2. revealed that out of the two hundred germplasm tested in which 116 germplasm/ varieties i.e. T-397, No7, No-11, Pol F-16, RLC-23, Polf-25, RLC-52, LC-2021, LCK-9303, LCK-9320, S-801, JLT-26, KL-134, LCK-9324, LCK-9436, EC-1398, EC-1402, EC-1497, EC-1352, EC-9204, EC-9828, EC-23595, EC322659, EC-322681, EC-199749, SJKO-6, SJKO-7, SJKO-18, SJKO-60, SJKO-62, SJKO-63, RSJ-29, RJK-20, NP (RR)-44, ES-1476, Barmer, ES-16381, GS-51, H-5, JLS293, JRF-3, POLF-23, 1406 LCK-87312, LCK-8722, No.294, 1420 LMH-21, NP-40, POLF-30, POLF-39, LC-

\begin{tabular}{|c|c|c|c|}
\hline S.No. & Wilting percentage & Scale & Abbreviation \\
\hline 1. & No wilting & Immune & I \\
\hline 2. & $0.1-10 \%$ & Resistant & R \\
\hline 3. & $10.1-25 \%$ & Moderately resistant & MR \\
\hline 4. & $25.1-50 \%$ & Moderately susceptible & MS \\
\hline 5. & $50.1-75 \%$ & Susceptible & S \\
\hline 6. & $75 \%$ and above & Highly susceptible & HS \\
\hline
\end{tabular}

Table 1: Rating scale was used for grouping of resistance and susceptibility to categories the germplam/varieties.

*Corresponding author: Mohit Kumar, Department of Plant Pathology and Entomology, Chandra Shekhar Azad University of Agriculture and Technology, Kanpur, India, Tel: 09760666300; E-Mail: mohitahlawat0666@gmail.com

Received: August 28, 2014; Accepted September 30, 2014; Published October 06, 2014

Citation: Kumar M, Tripathi UK, Tomer A, Kumar P, Singh A (2014) Screening of Linseed Germplasm for Resistance/Tolerance against Fusarium oxysporum F Sp. Lini (Bolley) Disease. J Plant Pathol Microb 5: 235. doi:10.4172/21577471.1000235

Copyright: @ 2014 Kumar M, et al. This is an open-access article distributed under the terms of the Creative Commons Attribution License, which permits unrestricted use, distribution, and reproduction in any medium, provided the original author and source are credited. 
Citation: Kumar M, Tripathi UK, Tomer A, Kumar P, Singh A (2014) Screening of Linseed Germplasm for Resistance/Tolerance against Fusarium oxysporum F Sp. Lini (Bolley) Disease. J Plant Pathol Microb 5: 235. doi:10.4172/2157-7471.1000235

\begin{tabular}{|c|c|c|c|c|c|c|c|}
\hline S. No. & Name of germplasm & Disease incidence & Scale & S. No. & Name of germplasm & Disease incidence & Scale \\
\hline 1. & EC-9826 & 17 & MR & 101. & EC-322646 & 9.25 & $\mathrm{R}$ \\
\hline 2. & EC-41656 & 9.28 & $\mathrm{R}$ & 102. & EC-822659 & 1.76 & $\mathrm{R}$ \\
\hline 3. & FR-3 & 17.50 & MR & 103. & EC-822681 & 2.49 & $\mathrm{R}$ \\
\hline 4. & Kanpur 41/2 & 9.58 & $\mathrm{R}$ & 104. & EC-199749 & 1.98 & $\mathrm{R}$ \\
\hline 5. & GS-204 & 25.49 & MS & 105. & SJKO-2 & 34.69 & MS \\
\hline 6. & GS-232 & 38.00 & MS & 106. & SJKO-6 & 2.76 & $\mathrm{R}$ \\
\hline 7. & GS-344 & 8.95 & $\mathrm{R}$ & 107. & SJKO-7 & 3.55 & $\mathrm{R}$ \\
\hline 8. & GS-362 & 14.37 & $\mathrm{R}$ & 108. & SJKO-10 & 26.66 & MS \\
\hline 9. & $\mathrm{H}-22$ & 8.14 & $\mathrm{R}$ & 109. & SJKO-18 & 2.69 & $\mathrm{R}$ \\
\hline 10. & $\mathrm{H}-42$ & 10.06 & MR & 110. & SJKO-22 & 7.00 & $\mathrm{R}$ \\
\hline 11. & HY-38 & 26.87 & MS & 111. & SJKO-25 & 14.77 & MR \\
\hline 12. & LCFR-7 & 29.46 & MS & 112. & SJKO-60 & 3.96 & $\mathrm{R}$ \\
\hline 13. & ILS-169 & 8.13 & $\mathrm{R}$ & 113. & SJKO-62 & 2.78 & $\mathrm{R}$ \\
\hline 14. & KL-31 & 41.00 & MS & 114. & SJKO-63 & 3.84 & $\mathrm{R}$ \\
\hline 15. & Jabalpur local & 13.6 & MR & 115. & RSJ-29 & 3.98 & $\mathrm{R}$ \\
\hline 16. & KL-176 & 8.0 & $\mathrm{R}$ & 116. & KL-225 & 13.00 & MR \\
\hline 17. & Kangra local & 5.47 & $\mathrm{R}$ & 117. & RKY-9 & 5.56 & $\mathrm{R}$ \\
\hline 18. & Kala-2 & 54.00 & $S$ & 118. & RKY-15 & 9.15 & $\mathrm{R}$ \\
\hline 19. & $\mathrm{KP}-4$ & 66.66 & $S$ & 119. & RJK-20 & 3.46 & $\mathrm{R}$ \\
\hline 20. & KP-13-13 & 17.20 & MR & 120. & NP (RR)-44 & 3.00 & $\mathrm{R}$ \\
\hline 21. & L-35 & 18.23 & MR & 121. & ES-1462 & 5.17 & $\mathrm{R}$ \\
\hline 22. & L-36 & 12.00 & MR & 122. & ES-1476 & 1.96 & $\mathrm{R}$ \\
\hline 23. & Mayur Bhanjan Local & 9.79 & $\mathrm{R}$ & 123. & Barner & 4.00 & $\mathrm{R}$ \\
\hline 24. & LCK-11 & 10.15 & MR & 124. & ES-16381 & 3.25 & $\mathrm{R}$ \\
\hline 25. & LCK-41 & 45.00 & MS & 125. & FRU-12 & 5.75 & $\mathrm{R}$ \\
\hline 26. & LCK-8504 & 11.30 & MR & 126. & C.F. Lalhi FC & 6.10 & $\mathrm{R}$ \\
\hline 27. & LCK-88311 & 9.25 & $\mathrm{R}$ & 127. & GS-51 & 4.20 & $\mathrm{R}$ \\
\hline 28. & LS-3 & 7.25 & $\mathrm{R}$ & 128. & Nagar Kot & 7.49 & $\mathrm{R}$ \\
\hline 29. & NP (RR)-18 & 7.64 & $\mathrm{R}$ & 129. & Kiran & 10.00 & $\mathrm{R}$ \\
\hline 30. & MS-3 & 48.11 & MS & 130. & EC-384154 & 8.44 & $\mathrm{R}$ \\
\hline 31. & MS-4 & 32.00 & MS & 131. & $\mathrm{H}-8$ & 14.33 & MR \\
\hline 32. & NCL-3512 & 10.50 & MR & 132. & $\mathrm{H}-10$ & 15.67 & MR \\
\hline 33. & T-397 & 3.00 & $\mathrm{R}$ & 133. & R-204 x4129 & 14.00 & MR \\
\hline 34. & No-7 & 2.96 & $\mathrm{R}$ & 134. & ECL-27 & 5.36 & $\mathrm{R}$ \\
\hline 35. & No-11 & 2.00 & $\mathrm{R}$ & 135. & $\mathrm{H}-43$ & 10.27 & MR \\
\hline 36. & No-16 & 6.59 & $\mathrm{R}$ & 136. & JRF-1 (8) & 5.39 & $\mathrm{R}$ \\
\hline 37. & No-18 & 13.10 & MR & 137. & GS-401 & 11.33 & MR \\
\hline 38. & No-22 & 9.50 & $\mathrm{R}$ & 138. & GS-407 & 12.52 & MR \\
\hline 39. & No-348 & 7.50 & $\mathrm{R}$ & 139. & $\mathrm{H}-5$ & 3.47 & $\mathrm{R}$ \\
\hline 40. & NP-19 & 8.53 & $\mathrm{R}$ & 140. & $\mathrm{H}-\mathrm{II}$ & 14.55 & MR \\
\hline 41. & NP-47 & 46.70 & MS & 141. & $\mathrm{H}-12$ & 13.10 & MR \\
\hline 42. & NP-26 & 6.57 & $\mathrm{R}$ & 142. & $\mathrm{H}-17$ & 15.12 & MR \\
\hline 43. & NP-115 & 37.80 & MS & 143. & $\mathrm{H}-15$ & 12.52 & MR \\
\hline 44. & NPHY-38 & 15.50 & MR & 144. & $\mathrm{H}-24$ & 35.20 & MS \\
\hline 45. & POLF-5 & 31.90 & MS & 145. & $\mathrm{H}-25$ & 8.40 & $\mathrm{R}$ \\
\hline 46. & POLF & 27.92 & MS & 146. & Meera & 14.35 & MR \\
\hline 47. & OP-2-2 & 28.15 & MS & 147. & ICAR-2 & 5.72 & $\mathrm{R}$ \\
\hline 48. & POLF-2 & 14.96 & MR & 148. & JLS-293 & 4.96 & $\mathrm{R}$ \\
\hline 49. & POLF-16 & 5.00 & $\mathrm{R}$ & 149. & JRF-3 & 2.55 & $\mathrm{R}$ \\
\hline 50. & POLF-17 & 8.75 & $\mathrm{R}$ & 150. & POLF-23 & 4.16 & $\mathrm{R}$ \\
\hline 51. & POLF-36 & 7.10 & $\mathrm{R}$ & 151. & KL-168 & 38.00 & MS \\
\hline 52. & RL-8-1 & 5.25 & $\mathrm{R}$ & 152. & KS-169 & 43.19 & MS \\
\hline 53. & RL-39-4 & 8.00 & $\mathrm{R}$ & 153. & KP-8 & 19.20 & MR \\
\hline 54. & RLC-23 & 3.96 & $\mathrm{R}$ & 154. & L-18 & 5.42 & $\mathrm{R}$ \\
\hline 55. & P-650 & 5.85 & $\mathrm{R}$ & 155. & $\begin{array}{c}\text { No.6 } \\
\text { LCK-87312 }\end{array}$ & 5.00 & $\mathrm{R}$ \\
\hline 56. & POLF-33 & 13.10 & MR & 156. & LCK-8722 & 4.54 & $\mathrm{R}$ \\
\hline 57. & RLC-3 & 7.00 & $\mathrm{R}$ & 157. & No.-294 & 4.36 & $\mathrm{R}$ \\
\hline 58. & RLC-7 & 6.49 & $\mathrm{R}$ & 158. & ES-44 & 39.10 & MS \\
\hline 59. & RLC-34 & 8.00 & $\mathrm{R}$ & 159. & $\mathrm{~L}-43$ & 36.79 & MS \\
\hline
\end{tabular}


Citation: Kumar M, Tripathi UK, Tomer A, Kumar P, Singh A (2014) Screening of Linseed Germplasm for Resistance/Tolerance against Fusarium oxysporum F Sp. Lini (Bolley) Disease. J Plant Pathol Microb 5: 235. doi:10.4172/2157-7471.1000235

Page 3 of 4

\begin{tabular}{|c|c|c|c|c|c|c|c|}
\hline 60. & OR-I-4 & 6.20 & $\mathrm{R}$ & 160. & LCK-152 & 37.15 & MS \\
\hline 61. & $\mathrm{~F}-42$ & 24.72 & MR & 161. & LCK-9436 & 21.00 & MR \\
\hline 62. & POLF-11 & 14.28 & MR & 162. & LCK-852 & 6.75 & $\mathrm{R}$ \\
\hline 63. & POLF-34 & 6.30 & $\mathrm{R}$ & 163. & $1420 \mathrm{MH}-21$ & 1.50 & $\mathrm{R}$ \\
\hline 64. & POLF-25 & 3.00 & $\mathrm{R}$ & 164. & $40-41-561$ & 34.12 & MS \\
\hline 65. & POLF-30 & 20.10 & MR & 165. & $40-41-562$ & 38.47 & MS \\
\hline 66. & R-552 & 5.75 & $\mathrm{R}$ & 166. & NP-40 & 5.00 & $\mathrm{R}$ \\
\hline 67. & RLC-52 & 3.43 & $\mathrm{R}$ & 167. & NP-65 & 31.20 & MS \\
\hline 68. & $5-93-3$ & 18.00 & MR & 168. & NP-66 & 26.19 & MS \\
\hline 69. & $5-91.35$ & 60.00 & $S$ & 169. & NP-71 & 6.54 & $\mathrm{R}$ \\
\hline 70. & Sagar Local & 7.15 & $\mathrm{R}$ & 170. & Rashrni & 22.73 & MR \\
\hline 71. & $4-6$ & 7.58 & $\mathrm{R}$ & 171. & NP-112 & 26.55 & MS \\
\hline 72. & $\mathrm{KL}-1$ & 11.20 & MR & 172. & NPHY-37 & 39.09 & MS \\
\hline 73. & LC-2014 & 6.00 & $\mathrm{R}$ & 173. & POLF-15 & 7.56 & $\mathrm{R}$ \\
\hline 74. & LC-2021 & 2.96 & $\mathrm{R}$ & 174. & POLF-129 & 10.25 & MR \\
\hline 75. & LC-2032 & 5.45 & $\mathrm{R}$ & 175. & PKY-2 & 11.00 & MR \\
\hline 76. & LC-2127 & 20.33 & MR & 176. & POLF-30 & 3.47 & $\mathrm{R}$ \\
\hline 77. & LC-2045 & 7.14 & $\mathrm{R}$ & 177. & POLF-39 & 4.10 & $\mathrm{R}$ \\
\hline 78. & LCK-9303 & 3.49 & $\mathrm{R}$ & 178. & NP (RR)-193 & 16.00 & MR \\
\hline 79. & LCK-9320 & 3.63 & $\mathrm{R}$ & 179. & 67-RLC-45 & 10.72 & MR \\
\hline 80. & RLC-55 & 6.92 & $\mathrm{R}$ & 180. & LC-2002 & 1.43 & $\mathrm{R}$ \\
\hline 81. & S-91-11 & 42.00 & MS & 181. & LC-2023 & 25.59 & MS \\
\hline 82. & S-91-25 & 7.33 & $\mathrm{R}$ & 182. & LC-2057 & 9.52 & $\mathrm{R}$ \\
\hline 83. & S-801 & 3.10 & $\mathrm{R}$ & 183. & S-91-26 & 18.61 & MR \\
\hline 84. & Sirmor-2 & 6.30 & $\mathrm{R}$ & 184. & KL-178 & 16.70 & MR \\
\hline 85. & Jabalpur-9 & 5.00 & $\mathrm{R}$ & 185. & LCK-9414 & 1.75 & $\mathrm{R}$ \\
\hline 86. & JLT-26 & 3.22 & $\mathrm{R}$ & 186. & RL-56-6-2 & 4.84 & $\mathrm{R}$ \\
\hline 87. & KL-134 & 3.10 & $\mathrm{R}$ & 187. & RL-903 & 23.15 & MR \\
\hline 88. & KL-168 & 44.00 & MS & 188. & KFS-11 & 14.92 & MR \\
\hline 89. & LC-2057 & 12.72 & MR & 189. & LCK-8504 & 9.15 & $\mathrm{R}$ \\
\hline 90. & LCK-9119 & 6.74 & $\mathrm{R}$ & 190. & SJKO-17 & 20.52 & MR \\
\hline 91. & LCK-9312 & 8.18 & $\mathrm{R}$ & & Chambal (SC) & 78.3 & HS \\
\hline 92. & LCK-9324 & 4.44 & $\mathrm{R}$ & 191. & SJKO-20 & 10.49 & MR \\
\hline 93. & LCK-9436 & 2.43 & $\mathrm{R}$ & 192. & SJKO-42 & 6.22 & $\mathrm{R}$ \\
\hline 94. & EC-1398 & 3.15 & $\mathrm{R}$ & 193. & EC-41590 & 4.24 & $\mathrm{R}$ \\
\hline 95. & EC-1402 & 1.43 & $\mathrm{R}$ & 194. & BRM-13 & 2.89 & $\mathrm{R}$ \\
\hline 96. & EC-1497 & 2.20 & $\mathrm{R}$ & 195. & S-91-25 & 5.00 & $\mathrm{R}$ \\
\hline 97. & EC-1352 & 2.49 & $\mathrm{R}$ & 196. & KL-217 & 10.29 & MR \\
\hline 98. & EC-9204 & 1.96 & $\mathrm{R}$ & 197. & $\mathrm{KL}-220$ & 12.55 & MR \\
\hline 99. & EC-9828 & 2.15 & $\mathrm{R}$ & 198. & $\mathrm{KL}-221$ & 4.45 & $\mathrm{R}$ \\
\hline \multirow[t]{2}{*}{100.} & EC-23595 & 1.66 & $\mathrm{R}$ & 199. & $\mathrm{KL}-223$ & 3.72 & $\mathrm{R}$ \\
\hline & & & & 200. & KL-227 & 7.84 & $\mathrm{R}$ \\
\hline
\end{tabular}

Abbreviation: R: Resistant, MR: Moderately Resistant, MS: Moderately Susceptible, S: Susceptible, HS: Highly Susceptible

Table 2: Reaction of linseed germplasm/varieties against Fusarium oxysporum f. sp. lini (2010-11).

2002, LCK-9414, RL-56-6-2, EC-41590, S-91-25, KL-221, KL-223, EC-41656, Kanpur 41/2, GS-344, H-22, ILS-169, KL-176, Kangra local, Mayerbhanj local, LCK88311, LS-3, NP (RR)-18, No.16, No22, No-348, No-19, NP-26, Polf-17, Polf-36, RL-8-1, RL-39-4, P-650, RLC-3, RLC-7, RLC-34, OR-1-4, Polf-34, R-552, Sagar local, 4-6, LC2014, LC-2032, LC-2045, RLC-55, S91-25, SIRMOR-2, LCK-9119, LCK9312, EC-322646, SJKO-22, Pky-9, Pky-15, ES-1462, FRW-12, C.F. Lalhi FC, Nagar Kot, EC-384154, ECL-27, JRF-1, H-25, ICAR-2, L-18, LCK-852, NP-71, POLF-15, LC-2057, LCK-8504, SJKO-42 and $\mathrm{KL}-227$ were found to be resistant to disease as the disease incidence in these cultivars were below 10 percent disease intensity. 51 cultures including some of the promising varieties like Kiran R-552 (EC-9826, FR-3, GS-362, H-42, Jabalpur local, KP-1313, L-35, L-36, LCK-11, LCK-8504, Rashmi, NCL-3512, No.-18, NPHY-38, POLF-2, POLF33, P-42, POLF-11, POLF-30, 5-93-3, KL-1, LC-2127, SJKO-25, KL225, H8, H-I0, R-204 x 4129, H-43, GS-401, GS-407, H-1l, H12, H-17,
H-15, Meera, KP-8, LCK-9436, POLF-129, RKY-2, NP (RR)-193, $67-$ RLC-45, S-91-26, KL-178, RL903, KFS-11, SJKO-17, SJKO-20, KL-217, JKL-220, KL229), fell in moderately resistant category. 30 genotypes i.e. GS-204, GS-232, HY-38, LCFR-7, KL-31, LCK-41, MS-3, MS-4, NP-47, NP-115, POLF-5, POLF-19, OP-2-2, S-9111, KL-168, SJKO2, H-24, KL-168, KU-169, L-43, LCK152, 40-41-561, NP-65, NP-66, NP-112, NPHY-37 and LC2023 were found moderately susceptible which were shown 10.1-25\% Disease intensity, 3 germplasm/ varieties were fond susceptible i.e. Kala-2, KP-4, 5-91-35 these germplasm were shown 25.1-50\% Disease intensity and the genotype 'Chambal' highly susceptible which shown $50-75 \%$ disease intensity.

The results revealed that out of the two hundred germplasm tested, 116 germplasm/varieties were found to be resistant to disease incidence in these cultivars was below 10 percent.

Use of resistant varieties is most economical, feasible and safe 
Citation: Kumar M, Tripathi UK, Tomer A, Kumar P, Singh A (2014) Screening of Linseed Germplasm for Resistance/Tolerance against Fusarium oxysporum F Sp. Lini (Bolley) Disease. J Plant Pathol Microb 5: 235. doi:10.4172/2157-7471.1000235

method to manage the disease. Keeping in view two hundred varieties/ germplasm of linseed were screened in wilt sick plot. Tested genotypes were categorized into resistant, moderately resistant, moderately susceptible, susceptible and susceptible as per incidence of wilt. However, some of the high yielding improved varieties reported [5] (Kerkhi et al.) to be resistant to wilt were fond moderately resistant and moderately susceptible in the present study.

The disease was characterized by rolling and withering of cotyledons when disease appeared at seedling stage, and young seedlings collapsed on ground. In grown up plants, dark green to brown patches appeared on leaves. The leaves shriveled later on dropping off symptoms from tip of the plant and drying of leaves and stem, discoloration of the vascular tissues were the similar symptoms to wilt caused by Fusarium oxysporum f. sp. Lini as reported by similar workers $[6,7]$.

\section{Conclusion}

A survey was conducted to know the occurrence and distribution of linseed wilt in Kanpur. The highest incidence (9\%) was recorded at Gopalpur (Bithoor) followed by Aliapur (Ghatampur) Kanpur. Overall wilt percentage was recorded about $(0-9 \%)$ in fields where linseed was not grown earlier and (10-37\%) in wilt sick field.
The screening of two hundred germplasm /varieties of linseed was carried out under artificial epiphytotic condition in the silt sick nursery. Out of which viz., 116 were found to be resistant, 51 were moderately resistant, 30 moderately susceptible and 3 were susceptible and 1 was highly susceptible as found in the study.

\section{References}

1. Broadfoot WC (1926) Studies on the parasitism of Fusarium lini Bolley. Phytopathology 16: 951-78.

2. Kerkhi SA, Srivastava RL, Singh Malik YP, Hussain K, Dubey SD, et al. (1997) Linseed; Technology for increased production.

3. Kommedahal T, Christensen JJ, Fredriksen RA (1970) A half century of research in Minnesota on flax wilt caused by Fusarium oxysporum. Tech Bull Agric Expt Stn 273: 35

4. Newkrik R (2008) Flax Feed Industry Guide. Flax Canada 2015

5. Rashid KY, Kenaschuk EO (1993) Effect of trifluralin on fusarium wilt in flax Can. J. Plant Sci. 73: 893-901.

6. Richardson OML (1979) Anonnoled list of seed borne diseases third. CM Phytopathology, 23: 320 .

7. Wilson EM (1944) Wilt disease of flax in Great Britain, Nature, London, 94 3918. 